\title{
Reformando la salud desde la prevención cuaternaria
}

\section{Enhancement of health quaternary prevention}

Correspondencia María Sofía Cuba Fuentes maria.cuba@upch.pe

Recibido: 04/03/2016 Arbitrado por pares

Aprobado: 27/04/2016

\section{Citar como:}

Cuba Fuentes MS, Morera González L. Reformando la salud desde la prevención cuaternaria. Acta Med Peru. 2016;33(1):65-9

\author{
María Sofía Cuba Fuentes 1,2, Lisdamys Morera González 2,3 \\ 1 Policlínico Juan José Rodríguez Lazo, EsSalud. Lima, Perú. \\ 2 Universidad Peruana Cayetano Heredia. Lima, Perú \\ 3 Puesto de Salud Jose Olaya, Ministerio de Salud. Lima, Perú.
}

\section{RESUMEN}

lan Mc Whinney define el papel del médico de familia/médico generalista como aquel ser científico y social con capacidades para observar la vida de los pacientes, definir con ellos la salud, aspiraciones y propósitos de los mismos. El ser individual nos da la posibilidad de encontrar un valor a la salud, tanto personal como colectiva, mirando desde las perspectivas de los determinantes sociales de la salud. El individuo en medio de este proceso necesita de un sistema que responda las necesidades de salud y determine un mejor estado de salud. En el siglo pasado nos enfrascamos en la búsqueda y análisis de factores de riesgo, y luego a enfrentarnos a ellos como causantes de las disfunciones individuales de nuestros pacientes. Así es como Marc Jamoulle, un médico de familia de Begium, nos trae los conceptos sobre la prevención cuaternaria, que ahora es parte del diccionario de WONCA: "La prevención cuaternaria es la acción tomada para identificar a un paciente en riesgo de sobretratamiento, para protegerlos de una nueva invasión médica, y sugerir a él / ella intervenciones, que son éticamente aceptable". Esta se relaciona directamente al accionar del médico de familia y la necesidad de pensar diferente. Y es esta la causa que nos mueve a que acojamos este modo de actuar mediante la prevención cuaternaria, como un modo de acción dentro de la atención primaria, pensando en nuestros pacientes y en el qué es mejor para ellos, aceptando nuestro límites reales.

Palabras clave:

Prevención cuaternaria; Atención primaria de salud; Medicina familiar y comunitaria (fuente: DeCS BIREME). 


\begin{abstract}
Ian McWhinney defines the role of the family doctor/general practitioner as that scientific and social professional with capabilities to observe the lives of patients, define their health, aspirations and aims. Being individuals gives us the chance to find a value for health, both personal and collective, looking from the perspective of the social determinants of health. The individual in the midst of this process requires a system that may fulfill the health needs and determines a better state of health. In the last century we engaged in searching and analyzing risk factors, and then facing them as causing the individual dysfunctions of our patients. That is how Marc Jamoulle, a family doctor from Belgium, brought the concept of quaternary prevention, which is now part of the WONCA dictionary: "Quaternary prevention is the action taken to identify a patient at risk of overtreatment, for protecting them from a new medical invasion, and to suggest him / her interventions which are ethically acceptable". This is directly related to the actions of the family doctor and the need to think differently. And this is the cause that moves us to allow us to accept this way of acting through quaternary prevention as a way of action within primary care, thinking of our patients and on what is best for them, accepting our real limitations.
\end{abstract}

Key words:

Quaternary prevention; Primary health care; Family practice (source: MeSH NLM).

\section{INTRODUCCIÓN}

La medicina familiar y comunitaria es una especialidad médica, pero también es una disciplina, con un cuerpo de conocimientos. El profesor lan Mc Whinney, describe los nueve principios que rigen las acciones del médico de cabecera. Uno de los principios descritos es que "los médicos de familia/médicos generalista ven cada contacto con sus pacientes como una oportunidad para la prevención de enfermedades y promoción de la salud" [1].

Debido a la importancia de las actividades preventivas en la práctica cotidiana y en los médicos de familia y comunidad, el concepto de salud, la normalidad y tipos de prevenciones serán discutidos en este ensayo.

\section{SALUD}

Hay muchas definiciones de la salud, incluida la de la Organización Mundial de la Salud (OMS): "La salud es un estado de completo bienestar físico, mental, social y no solamente la ausencia de enfermedad" [2].

Algunos autores como Stewart M. escriben acerca de la forma que los médicos tienen que pensar sobre la definición de salud, propusieron un concepto único para cada individuo que va más allá de la ausencia de enfermedad, abarca aspiraciones y propósitos de cada paciente [3].

Es importante para el clínico, evaluar, lo que significa la salud de forma individual para cada paciente. Las personas pueden tener valores que están en un nivel más alto que el "valor de la salud". El concepto de salud puede estar influenciado por los contextos proximal y distal así como también por los valores personales [4].

La salud puede ser considerada como el resultado de la interacción de algunos factores determinantes, como la educación, los ingresos, la vivienda, el trabajo decente y las condiciones más básicas, como el acceso al agua potable, condiciones que aún no se alcanzan a toda la población en muchos países [5].

La existencia de un sistema de salud que responda adecuadamente a las necesidades de las personas es también un factor determinante en la salud de las personas, familias y comunidades.

\section{EL SIGNIFICADO DE LA NORMALIDAD}

El concepto de normalidad mucha veces es un punto de corte arbitrario que se decide por expertos o estudios que indican desde cuando puede haber más riesgo para la salud. El diagnóstico de hispertensión diabetes u osteoporosis, se basa en curvas de normalidad. Esta normalidad muchas veces no toma en cuenta el punto de vista del paciente; el ser catalogado como anormal puede beneficiarlo pero también puede ser el inicio de una serie de intervenciones que no tendrán un beneficio importante y tal vez puedan generar daño $[6,7]$.

La aspiración a la normalidad es común; la aspiración de la normalidad en la salud es también un valor de lograr. Para identificar a los individuos en riesgo requiere una comprensión de la prevalencia de algunos problemas en la comunidad y reconocer de variabilidad humana [8].

La presencia de un factor de riesgo aumenta la posibilidad de presentación de la enfermedad; sin embargo debemos señalar que los factores de riesgo no son factores causales y no son suficientes para el desarrollo de la enfermedad.

Las prácticas preventivas sumadas a factores de riesgo tienen un valor más alto y se exigen mucho más en los individuos más jóvenes, más educados y más ricos. Esta población suele 


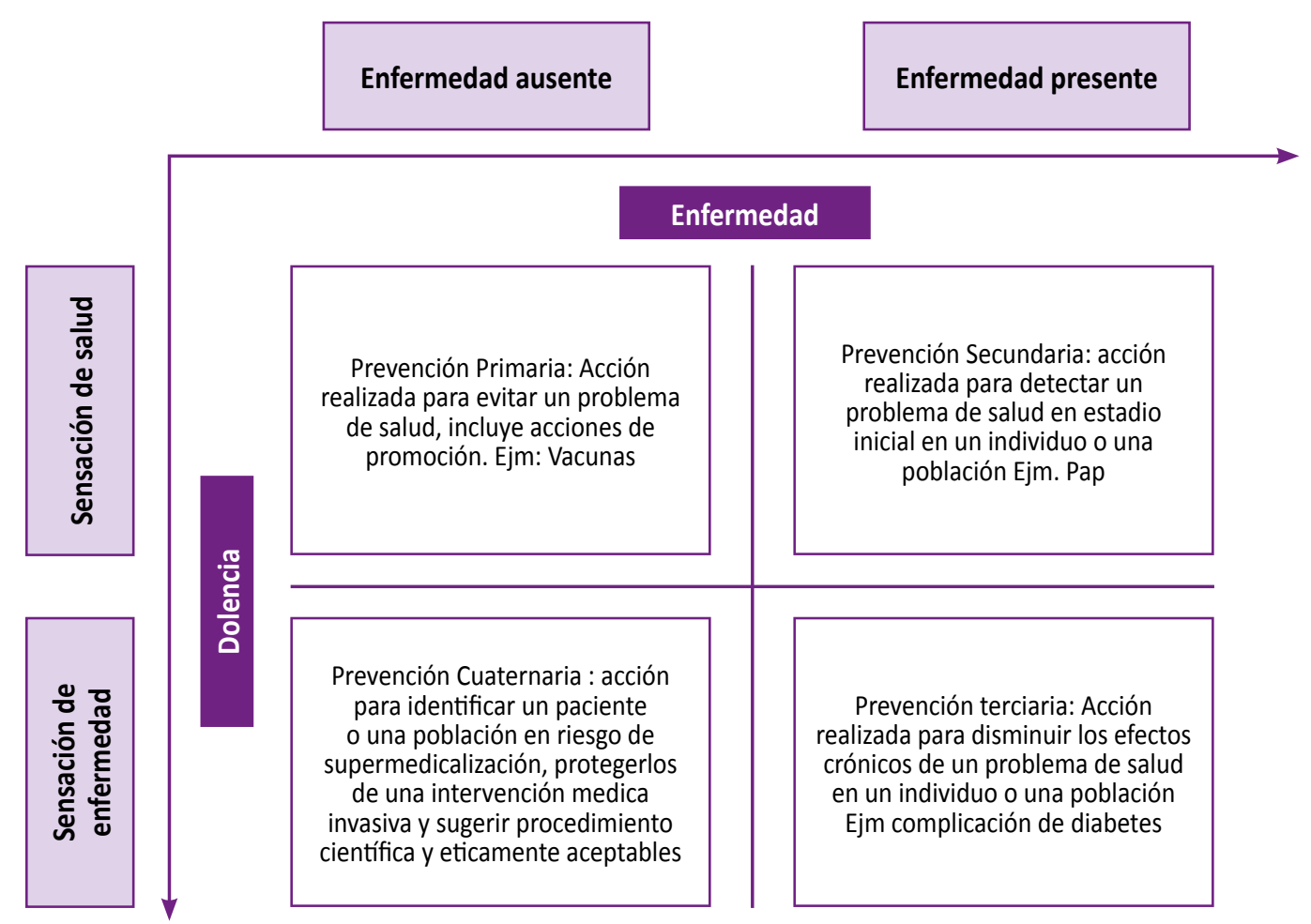

Adaptado de Gusso G. Tratado de Medicina de Familia e Comunidade 2012

Figura 1. Cuatro dominios para la prevención en la practica clínica

ser menos vulnerables y menos propensos a desarrollar la enfermedad; paradójicamente reciben más intervenciones preventivas [9].

A menudo, el tiempo y los recursos del sistema de salud innecesarios se gastan en actividades de prevención en individuos de bajo riesgo, a expensas de las actividades curativas en los complejos con múltiples comorbilidades, que terminan recibiendo menos atención o la falta de acceso al sistema de salud.

El concepto de normalidad y la prevención de los factores de riesgo podrían afectar al equilibrio del sistema y la atención adecuada a las personas.

\section{LA PREVENCIÓN Y LA PREVENCIÓN CUATERNARIA}

La práctica de la medicina de alguna forma implica un contrato social con la población, por muchos años este contrato estuvo relacionado a curar, sanar, acompañar en el sufrimiento; sin embargo en los últimos años se sumó un nuevo rol, el de prevenir enfermedades; una tarea que depende no sólo de los prestadores sino tal vez mucho más importante de las condiciones de vida de las personas [10].
Prácticas, como inmunizaciones o promoción de estilos de vida saludables, han demostrado muchos beneficios para la salud de la población, pero no todas las actividades de prevención son las mismas, algunos de ellos tienen consecuencias potenciales, incluyendo: una posible interrupción de las capacidades culturales e individuales para hacer frente a la enfermedad, el dolor y la muerte; aumentó el miedo y la percepción de la enfermedad cuando no existen; y la frustración de los clínicos a través de una creciente lista de requisitos que son imposibles de acomodar dentro de la visita clínica. Pasando de un "contrato curativo" a un "contrato preventivo" [11].

Leavell y Clark acuñaron los términos, prevención primaria, secundaria y terciaria al final de 1940. Estos términos se han utilizado ampliamente en la literatura médica y se han utilizado generaciones de enseñanza de los estudiantes de medicina. Los conceptos desarrollados por Leavell y Clark, tienen una vista línea de tiempo, antes y después del problema de salud; que se centra en la enfermedad, la clasificación se basa en la aparición de la enfermedad y su complicaciones [12].

Basado en el trabajo de lan McWhinney definir el método clínico para la medicina de familia; Marc Jamoulle, un médico de familia de Begium elaboró el concepto de la prevención cuaternaria, que ahora es parte del diccionario de WONCA:

"La prevención cuaternaria es la acción tomada para identificar a un paciente en riesgo de sobretratamiento, para protegerlos 
de una nueva invasión médica, y sugerir él / ella intervenciones, que son éticamente aceptable" [13].

Jamoulle indicó que muchas de las acciones de la práctica diaria de un médico de familia están relacionadas con la práctica de la prevención cuaternaria.

Describió los 4 tipos de prevención en un gráfico de $2 \times 2$ (Figura 1), la enfermedad y la dolencia en los lados; y afirmó que en cada encuentro aplicamos más de un tipo de prevención:

- Cuando una persona no tiene la dolencia ni la enfermedad, debemos aplicar la prevención primaria.

- Cuando una persona no tiene la dolencia pero tiene la enfermedad, debemos aplicar la prevención secundaria.

- Cuando una persona tiene la enfermedad y la dolencia debemos que aplicar la prevención terciaria.

- Cuando una persona tiene la dolencia pero no la enfermedad debemos aplicar la prevención cuaternaria.

Para la práctica de la medicina familiar, es importante analizar, ¿qué es una buena práctica?; ¿Cómo podemos evitar hacer daño a nuestros pacientes?

En los últimos 30 años, la tecnología ha mejorado el diagnóstico y el tratamiento de muchas enfermedades. Pero un nuevo problema planteado, las fuerzas del mercado están influyendo cada vez más sistemas médicos y las prácticas de salud. Los nuevos conocimientos sobre la estratificación del riesgo en algunos casos, hace que un gran número de personas entran en la categoría de la enfermedad [14].

A veces, la clasificación de la enfermedad se adapta a la necesidad de la industria, y la división entre lo normal y no es normal a veces no está claro.

Se espera que los médicos de familia desarrollen una nueva forma de pensar acerca de los beneficios y los daños potenciales de la prevención en la práctica clínica.

Algunas de las acciones de la práctica diaria de un médico de familia están relacionadas con la práctica de la prevención cuaternaria. Por ejemplo, la necesidad real de hacer "chequeos" en las personas sanas, nuevos tratamientos farmacológicos más caros, pero no es mejor que lo habitual en muchas enfermedades, las acciones de salud basadas en el conocimiento científico y, las expectativas del paciente. Podemos decir citando a Marc Jamoulle que: "La prevención cuaternaria es una manera de entender y cuestionar los límites del trabajo médico de familia".

La prevención cuaternaria, abarca las consecuencias del encuentro entre la expectativa del paciente y la incertidumbre del médico, podemos decir entre la dolencia y la enfermedad.

La decisión médica es en parte racionalizada y explícita, sino que también depende de la psicología del médico, en parte implícita (debido a la contingencia de modelos y valores del médico) y se elabora de acuerdo a una lógica bayesiana en un contexto de incertidumbre [15].

Los médicos de familia se ocupan de los pacientes, las poblaciones de todo tipo no seleccionados y entre los cuales la prevalencia de una enfermedad específica es en general baja. Los médicos de espacialidades secundarias se ocupan de poblaciones de pacientes entre los que se espera que la prevalencia de problemas de salud específicos es alta [16].

Las intervenciones médicas basadas en el mejor conocimiento científico disponible y las preferencias del paciente: la medicina y la calidad en los servicios de salud basada en la evidencia; son componentes muy importantes de la práctica de la prevención cuaternaria [17]

La definición de la prevención cuaternaria contiene varios otros conceptos que son ya sea compleja o sutil: el concepto de "riesgo", el concepto de "exceso de tratamiento / diagnóstico excesivo", el concepto de "protección del paciente", el concepto de "invasión médica" y finalmente, el concepto de "éticamente aceptable" [18].

Cada uno de estos conceptos son difíciles de medir y es la firma de un límite del conocimiento. Los cinco conceptos; "Riesgo / sobre / protección / invasión / aceptable" están sujetos a interpretaciones dependiendo de las opciones económicas o ideológicas y sobre el conocimiento científico aceptable [19].

\section{LA PREVENCIÓN CUATERNARIA COMO UN CAMPO DE INTERVENCIÓN}

Las prácticas de prevención cuaternaria, están muy relacionadas con la relación médico paciente, porque incluye todas las intervenciones que los médicos de familia hacen que no dañan, para controlar la ansiedad o para hacer frente a la falta de conocimiento o certeza acerca de los problemas de salud, tanto en el médico como en el paciente [20].

Los estudiantes de medicina, residentes y médicos de familia tienen que aprender a lidiar con preocupaciones de los pacientes y controlar sus propias dudas. Los sistemas de salud deben implementar medidas para garantizar la calidad, como la aplicación de la mejor evidencia disponible para la toma de decisiones compartidas con el paciente e identificar los peligros de sobrediagnóstico y sobretratamiento y la mercantilización de algunas enfermedades [21].

El médico de familia debe usar su conocimiento íntimo de la persona y su entorno para ofrecer actividades preventivas en el marco de las características, las creencias y la salud situación de las personas que atiende.

Fuente de financiamiento: Autofinanciado Conflictos de interés: Los autores declaran no tener conflictos de interés con la publicación de este artículo 


\section{REFERENCIAS BIBLIOGRÁFICAS}

1. McWhinney I. Principles of family medicine. In: McWhinney I. Textbook of family medicine. New York: Oxford University Press Inc.; 2009. p. 13-28.

2. World Health Organization (WHO). Who definition of Health Preamble to the Constitution of the World Health Organization as adopted by the International Health Conference, New York, 19-22 June, 1946; signed on 22 July 1946 by the representatives of 61 States (Official Records of the World Health Organization, no. 2, p. 100) and entered into force on 7 April 1948.

3. Stewart M, Brown JB, Weston WW, McWhinney IR, McWilliam CL, Freeman TR. Patient-Centered Medicine: Transforming the Clinical Method. 3rd Edition. Oxford UK: Radcliffe Publishing Ltd.; 2014.

4. McWhinney I. The enhancement of health and the prevention of disease. In: McWhinney I. Textbook of family medicine. New York: Oxford University Press Inc.; 2009. p. 193-216.

5. World Health Organization (WHO). The Social determinants in Health. 2nd Edition Copenhagen, Denmark: WHO; 2003.

6. Gervas. J, Pérez Fernández M. Sano y salvo y libre de intervenciones sanitarias innecesarias. Barcelona: Los libros del Lince; 2013.

7. Rose G. Sick individuals and sick populations. Int J Epidemiol 2001;30(3):427-32.

8. Illich I. Medical Nemesis. The expropriation of health. New York: Pantheon Books; 1976.

9. Gervas J. Protecao dos pacientes contra os excesso e danos das actividades preventivas. En: Gusso G. Tratado de Medicina de familia e comunidade: Principios de formacoa e Practica. Porto Alegre: ArtMed; 2012. p 212-20.

10. Sackett DL. The arrogance of preventive medicine. CMAJ $2002 ; 167(4): 363-4$.
11. Moynihan R, Smith R. Too much medicine? BMJ. 2002;324(7342):85960.

12. Leavell $\mathrm{H}$, clak $\mathrm{E}$. Preventive Medicine for the doctor in his community an epidemiologic aproach. New York: Mc Graw-Hill; $195^{8}$.

13. Bentzen N. WONCA Dictionary of General/Family Practice. Copenhagen, Denmark: Laegeforeningens Forlag; 2003.

14. Jamoulle M. Prevencao Quaternaria: primero nao causar dano. En: Gusso G. Tratado de medicina de familia e comunidade: Principios de formacoa e Practica. Porto Alegre: ArtMed; 2012. p. 205-11.

15. Kuehlin T, Sghedoni D, Visentin G, Gérvas J, Jamoulle M. Quaternary prevention: a Task for general practitioner. Primary Care. 2010;10(18):350-4.

16. McWhinney I. Illnes in the Community. In: McWhinney I. Textbook of family medicine. New York: Oxford University Press Inc.; 2009. p. 30-8.

17. Melo M. A prevencao qauternaria: Contra excessos da medicina. Rev Port Clin Geral. 2007;23:289-92.

18. Jamoulle M. The four duties of family doctors: quaternary prevention - first, do no harm. HK Pract. 2014;36(2):72-7.

19. Jamoulle M, Bernstein J, Pizzanelli Báez M, Da Silva A, Wagner $\mathrm{H}$. Prévention quaternaire: un concept fondé sur le temps et la relation. Première partie. Un test improbable: la connaissance de quelques-uns contre le doute de la plupart. Médecine. 2014;10(1):24-7.

20. Jamoulle M, Gomes LF. Prevenção Quaternária e limites em medicina. Rev Bras Med Fam Comunidade. 2014;9(31):186-91.

21. Nève J, Jamoulle M, Terra MA, Bernstein J. Prevencion cuaternaria, una tarea explicita del médico generalista. SER MÉDICO [Internet]. 2014;(14) [citado el 12 de marzo de 2016]. Disponible en: http:// www.smu.org.uy/publicaciones/sermedico/2014/sm14/dossier.pdf

\section{Las ediciones anteriores de Acta Médica Peruana están disponibles en:}

\section{www.scielo.org.pe}

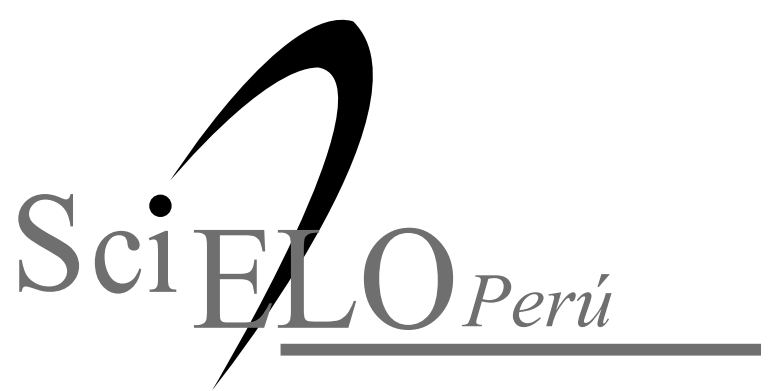

